\title{
Exploring Critical Success Factors for Blockchain-based Intelligent Transportation Systems
}

\author{
Murat Tahir Çaldağ a ${ }^{*}$, Ebru Gökalp ${ }^{\text {a }}$ \\ ${ }^{a}$ Faculty of Economics and Administrative Sciences, Department of Technology and Information Management, Baskent University, Ankara, Turkey
}

\begin{abstract}
Utilization of Intelligent Transportation Systems (ITS) provides increasing demands on decreasing traffic congestions, transportation safety, and environmental problems as well as increasing transportation capacity. Over the past few years, one of the most disruptive and transformational technologies, blockchain technology, has emerged. As a result of offering more security, privacy, traceability, transparency, and decentralized authority, the utilization of blockchain technology in ITS have increased drastically. Despite this increase, there is a lack of a good understanding of what are the Critical Success Factors (CSF) to support IT providers for designing their products properly, and to provide industry leaders to focus on the main drivers of their outstanding projects. This study aims to explore and analyze CSFs that can facilitate the success of blockchain-based ITS, which has so far been neglected despite its remarkable relevance. In doing so, eleven primary studies, identified as a result of conducting a systematic literature review (SLR), were taken as a baseline to develop the model of CSFs. Additionally, since the number of existing studies related to CSFs for blockchain and ITS are limited, an expert panel was formed to evaluate and contribute to the model. The developed model of CSF for blockchain-based ITS, having a comprehensive approach, consists of 29 CSFs defined under five main contexts, namely technical, management, governance, quality of service, and quality of life.
\end{abstract}

\section{Keywords:}

Blockchain;

Intelligent Transportation Systems;

Critical Success Factors.

\section{Article History:}

$\begin{array}{llll}\text { Received: } & 30 & \text { June } & 2020 \\ \text { Accepted: } & 03 & \text { October } & 2020 \\ \text { Published: } & 13 & \text { October } & 2020\end{array}$

\section{1- Introduction}

The population living in cities is increasing day by day. Accordingly, the increasing number of vehicles causes traffic congestion problem in cities. Alternative, complementary solutions to tackle this problem are implementing new transportation policies, such as restriction of private cars on busiest thoroughfares, increasing road capacities, such as widening the existing roads or constructing new roads, or optimizing the use of the existing transportation system by utilization of Intelligent Transportation Systems (ITS). Since many cities were built a long time ago, their land resources to widen the roads or construct new roads, highways, and freeways are so limited and costly. However, utilization of ITS provide a decreased traffic congestion rate, causing an increase in air pollution, fuel consumption, the risk of accident, and the stress level of the human [1-3].

ITS are developed based on the implementation of integrated information and communications technologies in the field of transportation systems. They provide integration of vehicles, roads, and people by utilization of advanced information and communication technologies, such as Radio-Frequency Identification (RFID) scanners and Global Positioning Systems (GPS) for traffic data collection, Advanced Traveler Information Systems (ATIS) for easing travel decisions, Advanced Management Systems (AMS) for obtaining control on transportation systems, connected vehicles, electric vehicles, and intelligent traffic systems $[1,4]$.

\footnotetext{
* CONTACT: Mtcaldag@baskent.edu.tr

DOI: http://dx.doi.org/10.28991/esj-2020-SP1-03
}

(C) 2020 by the authors. Licensee ESJ, Italy. This is an open access article under the terms and conditions of the Creative Commons Attribution (CC-BY) license (https://creativecommons.org/licenses/by/4.0/). 
These technological advancements bring not only more convenient transportation services but also significant issues as privacy, security, trust, and complexity. In order to maintain the ITS ecosystem in a profitable, stable, effective, and secure manner, it is critical to establish trusted cooperation and to develop a secure, trusted, and decentralized architecture. In order to satisfy this necessity, blockchain technology provides excellent solutions.

Blockchain technology first came out in 2008 in the cryptocurrency market. It provides a peer-to-peer (P2P) electronic cash transaction platform with chronological, encrypted immutable, and chained blocks. It provides promising solutions, including decentralized management, immutable audit trail, privacy, security, data provenance, the decreased total cost of ownership, and robustness [5]. After realizing how versatile it is, blockchain technology has recently attracted intense attention from both industry and academia [6]. According to Deloitte [7], blockchain will become one of the top five strategic priorities for the next two years.

Critical Success Factors (CSFs) can be defined as characteristics, factors, conditions, or variables that have a significant impact on the success of a business [8], and they must go right for achieving successful results. CSFs can be used during the strategy development process on environmental analysis, resource analysis, and strategy evaluation. These factors need to be carefully managed, maintained, controlled for the expected success for different sectors as effective advertisement, new product development, cost efficiency, technological competence, adequate production capacity [8]. CSFs are used to measure the success quantitively to manage it more effectively. As asserted in the quality management domain, what gets measured gets managed.

As a result of the literature review, it was observed that the limited number of studies unifies blockchain with ITS since blockchain is at an infancy stage in its development. Additionally, the existing studies mainly concentrate on providing a solution for particular use-cases and examining the benefits and challenges of blockchain in ITS. Neither industry nor academia has analyzed the CSFs of blockchain-based ITS. Thus, it can be asserted that there is a need to investigate the CSFs of blockchain-based ITS to manage it efficiently and effectively from a holistic point of view. Correspondingly, this study aims to satisfy this gap by developing a model of CSFs for blockchain-based ITS to guide system developers and industry leaders on how to develop a successful blockchain-based ITS.

The remainder of the paper is structured as follows: first, the background of the study is given, followed by the results of the systematic literature review (SLR) related to CSFs of blockchain-based ITS. Then, the proposed model of CSFs for evaluating blockchain-based ITS are given, and CSFs are described. Lastly, the conclusion of the study is presented.

\section{2- Background of the Study}

ITS use analyzed data collected from various sources, such as sensors, mobile cellular networks, meteorological sensors, seismic sensors, cameras, GPS based receivers, microwave detectors, inductive loop detectors. They consist of six fundamental components as advanced transportation management systems, advanced traveler information systems, advanced vehicle control systems, business vehicle management, advanced public transportation systems, advanced urban transportation systems [9].

Blockchain, defined as a decentralized ledger system that uses cryptographically chained blocks for data transfer, verifies the data through a peer-to-peer (P2P) network $[5,10,11]$. Each transaction is verified by a group of nodes maintained by the majority of the participants in the system $[5,12]$. Blockchain has a revolutionary effect not only on the finance sector with cryptocurrency and decentralized authority but also on other sectors as manufacturing, healthcare, or supply chain management, owing to new ways of more secure and transparent transactions [5, 13-15]. The benefits of blockchain technology can be described as distributed consensus, anonymity [12], transparency, accountability, interoperability, financial effectiveness, collaboration, agility [5], privacy, security, and scalability [15].

As a result of offering these benefits, blockchain-based ITS applications are attracted intense attention from both academicians and practitioners, and several blockchain-based ITS applications and prototypes have been developed. Some of the successful applications of blockchain-based ITS are carpooling applications, traffic control and management systems, and vehicular ad-hoc networks, as described below.

\section{2-1-Blockchain-based Carpooling Applications}

Blockchain-based carpooling applications, as La'Zooz [16], CoRide, and FICA [17] have a goal of building a worldwide, decentralized, private, anonymous, and auditable ride-sharing network to optimize empty seats and unused cargo spaces [11]. One of the main distinctive features of these applications from other ride-sharing networks like Uber is the decentralized authority. Another blockchain-based ITS application is the CarbonCount [18], which is developed to provide the calculation of individual carbon footprint based on analysis of personal mobility data to convince society to use cleaner transportation options and reduce carbon dioxide emissions. 


\section{2-2-Blockchain-based Traffic Control and Management Systems}

There are also some examples of blockchain-based traffic control and management systems, as Semi-centralized Traffic Signal Control (SCTSC) [19], which aims to support the automation of traffic signaling to enhance the efficiency of traffic control and management. Utilization of blockchain technology on the traffic control systems provides deciding traffic signal timing based on the data analysis and the results of voting by the community. Another blockchain-based ITS is a prototype of a toll road system developed by Oaken Innovations [20].

\section{2-3-Blockchain-based Vehicular Ad-Hoc Networks}

Chorus Mobility is a decentralized system that combines vehicular ad-hoc networks (VANET) and Ethereum to provide services and enforce rules on the smart transportation system [21]. Another study proposes a self-managed VANET system for enabling decentralization [22]. The utilization of blockchain technology on the VANET system removes the need for third parties and the need for human interaction as well as prevents changing the transaction agreements. Blockchain-based intelligent vehicles provide to remove challenges related to authentication, trust, and validation on vehicular communication [23]. For example, one of the proposed models, the Intelligent Vehicle-Trust Point (IV-TP), provides incentives, fast and secure communication between self-driven cars [23].

Although blockchain-based ITS are attracted intense attention from both academia and industry, it is seen that there is a lack of a good understanding of what are the CSF for blockchain-based ITS to focus on the main drivers of projects. This study aims to explore and analyze CSFs that can facilitate the success of blockchain-based ITS. The following section includes the research methodology followed while exploring these CSFs.

\section{3- Research Methodology}

In this section, the following research methodology and the development stages of the model of CSFs are described in detail. The research methodology conducted in this study consists of two phases, the literature review of existing studies and the development of the model of CSFs for blockchain-based ITS.

\section{3-1-Systematic Literature Review}

Existing studies regarding the CSFs of blockchain and ITS were identified by following the SLR method proposed by Kitchenham [24]. SLR method includes three main phases, including planning, executing, and reporting the results of the SLR process.

\section{3-1-1- Phase - I: Planning of the SLR Process}

In this phase, The SLR process focuses on developing a review protocol to define the objective(s) and Research Questions (RQs) of the SLR process, to establish a basis to identify proper databases, and to develop a research strategy. The steps of the SLR process are given in Figure 1. The principal aims are to examine the existing studies related to the CSFs for blockchain-based ITS. Two main RQs, determined based on these aims, are as follows:

- RQ1: What are the existing studies related to the CSFs for blockchain-based ITS?

- RQ2: What are the main factors affecting the success of blockchain-based ITS?

\section{3-1-2- Phase - II: Conducting the SLR Process}

Following the definition of planning and review protocols, the review was performed in July 2020, according to the steps given in Figure 1. In this review process, the following query was used to collect matching studies in title, abstract, or keywords; ("blockchain") AND ("intelligent transportation systems" OR "smart mobility" OR "smart transportation") AND ("critical success factors" OR "success factors" OR "evaluation factors" OR "assessment factors" OR "quality factors") by examining the Scopus and Web of Science databases. The result of the preliminary search provided that there is no matching studies on existing literature. Since there is no study related to the CSFs of blockchain-based ITS, we conducted two separate SLRs for determining the CSFs of blockchain technologies and ITS for the research objectives.

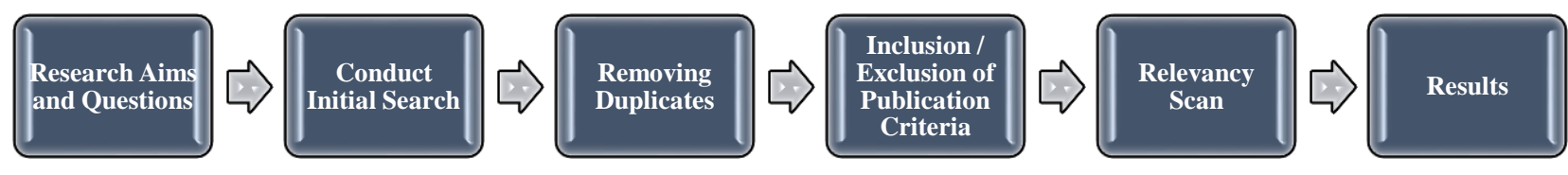

Figure 1. Systematic Literature Review Process. 


\section{The SLR Process for CSFs of Blockchain Technology}

The first review process covers to search the existing literature on the CSFs of blockchain technology. The following query was used to collect relevant studies in title, abstract or keywords; ("blockchain") AND ("critical success factors" OR "success factors" OR "evaluation factors" OR "assessment factors" OR "quality factors") by examining the Scopus and Web of Science databases. Inclusion and exclusion criteria were defined to evaluate the relevance of the studies according to established research questions. English language and journals with the SSCI and SCI indexes, book chapters, as well as conference proceedings were covered. However, other publications, which include series, meetings, whitepapers, and review papers, were excluded in the scope of this SLR process. Initially, 45 studies were identified. After removing the duplicates and exclusion of series and reviews, a total of 21 studies remained. After evaluating these studies, according to their abstracts, titles, and keywords, a total of nine related studies remained. After reading these nine studies fully, it was realized that all of them did contribute to defining the CSFs for blockchain, and the nine studies were labeled as primary studies. The number of papers respective to the SLR steps is given in Figure-2, and the primary studies investigating the CSFs of blockchain technology are given in the Appendix I.

\section{The SLR Process for CSFs of ITS}

The second review process was performed to search the existing literature on the CSFs of ITS. The following query was used to collect relevant studies in title, abstract or keywords; ("intelligent transportation systems" OR "smart mobility" OR "smart transportation") AND ("critical success factors" OR "success factors" OR "evaluation factors" $O R$ "assessment factors" OR "quality factors") by examining the Scopus and Web of Science databases. Inclusion and exclusion criteria were defined to evaluate the relevance of the studies according to established research questions. English language and journals with the SSCI and SCI indexes, book chapters, as well as conference proceedings were covered. However, other publications, which include series, meetings, whitepapers, and review papers, were excluded in the scope of this SLR process. Initially, 12 studies were identified. After removing the duplicates and exclusion of series and reviews, a total of 10 studies remained. After evaluating these studies, according to their abstracts, titles, and keywords, a total of two related studies remained. After reading these two studies fully, it was realized that all of them did contribute to defining the CSFs of ITS. Therefore, these studies were labeled as primary studies, as seen in Figure 2. The details of the primary studies, investigating the CSFs of ITS, are given in the Appendix I.
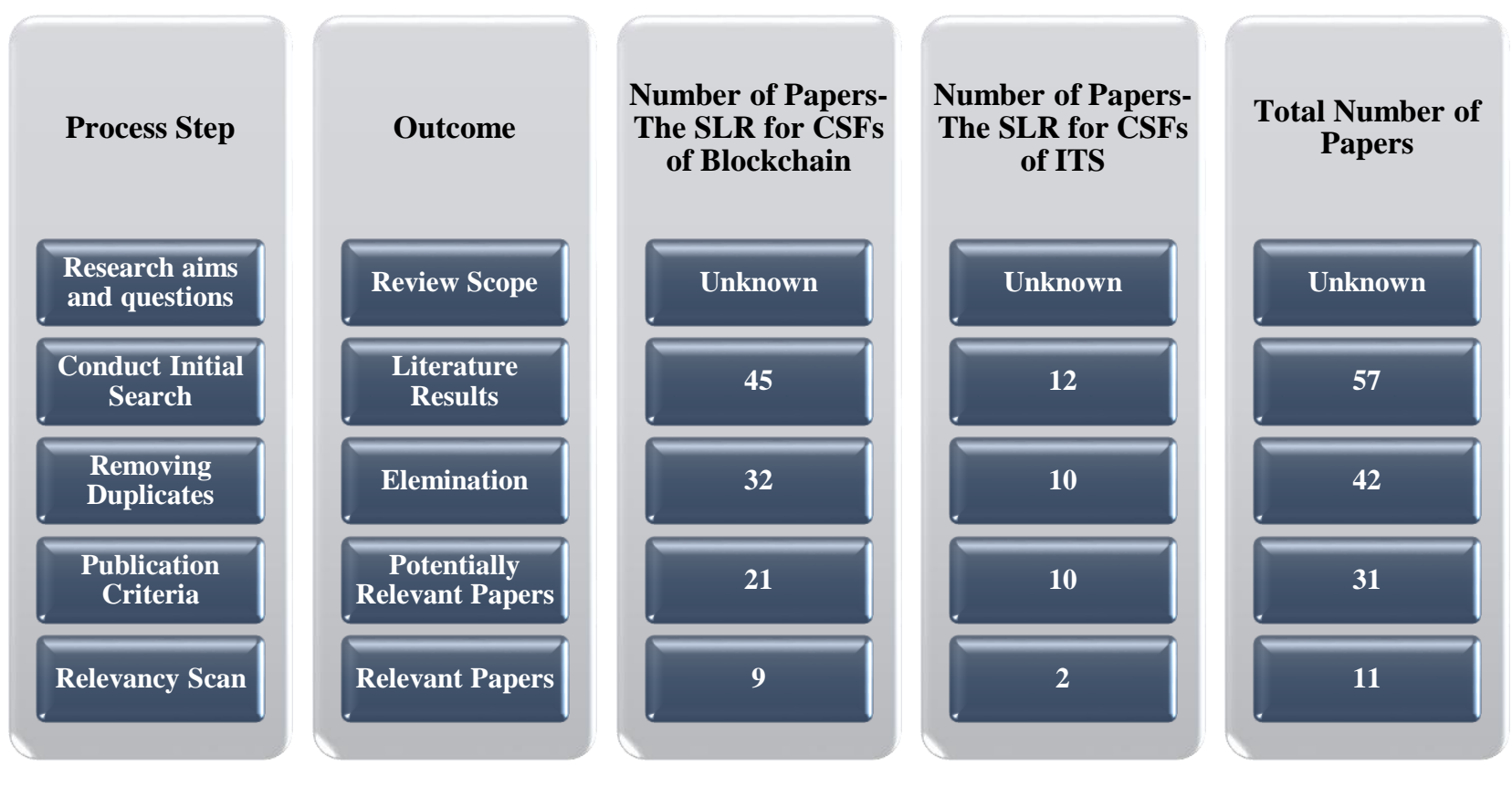

Figure 2. The Systematic Literature Review Results.

\section{3-1-3- Phase - III: Examining Research Results}

Eleven primary studies, exploring the significant CSFs of blockchain and ITS, were identified as a result of the SLR, as given in the Appendix I. Since blockchain and ITS are novel technologies, they are still at their infancy, the number of studies aiming to investigate determinants of success of blockchain and ITS are limited, and there is no study aiming to investigate the CSFs of blockchain-based ITS. Correspondingly, this study aims to bridge this gap by developing a model of CSFs for blockchain-based ITS. The developed model is described in the next section. 


\section{4- The Proposed Model of CSFs for Blockchain-based ITS}

The eleven primary studies, identified as a result of the SLR, were taken as a baseline to develop the model of CSFs. Additionally, since the number of studies related to CSFs for blockchain-based ITS are zero and there are a limited number of studies for CSFs for blockchain and ITS, separately, the expert panel comprising senior academicians, senior IT managers, and senior executive members working in the Blockchain and ITS domains was formed to evaluate and contribute the model of CSFs. The CSFs were discussed through a series of meetings conducted to reach a consensus. Finally, they reached a consensus to develop the model of CSFs, as given in Figure 3. The CSFs are classified into five main dimensions/contexts of Technical, Management, Governance, Quality of Life, and Quality of Service.

\section{Blockchain-based Intelligent Transportation Systems}

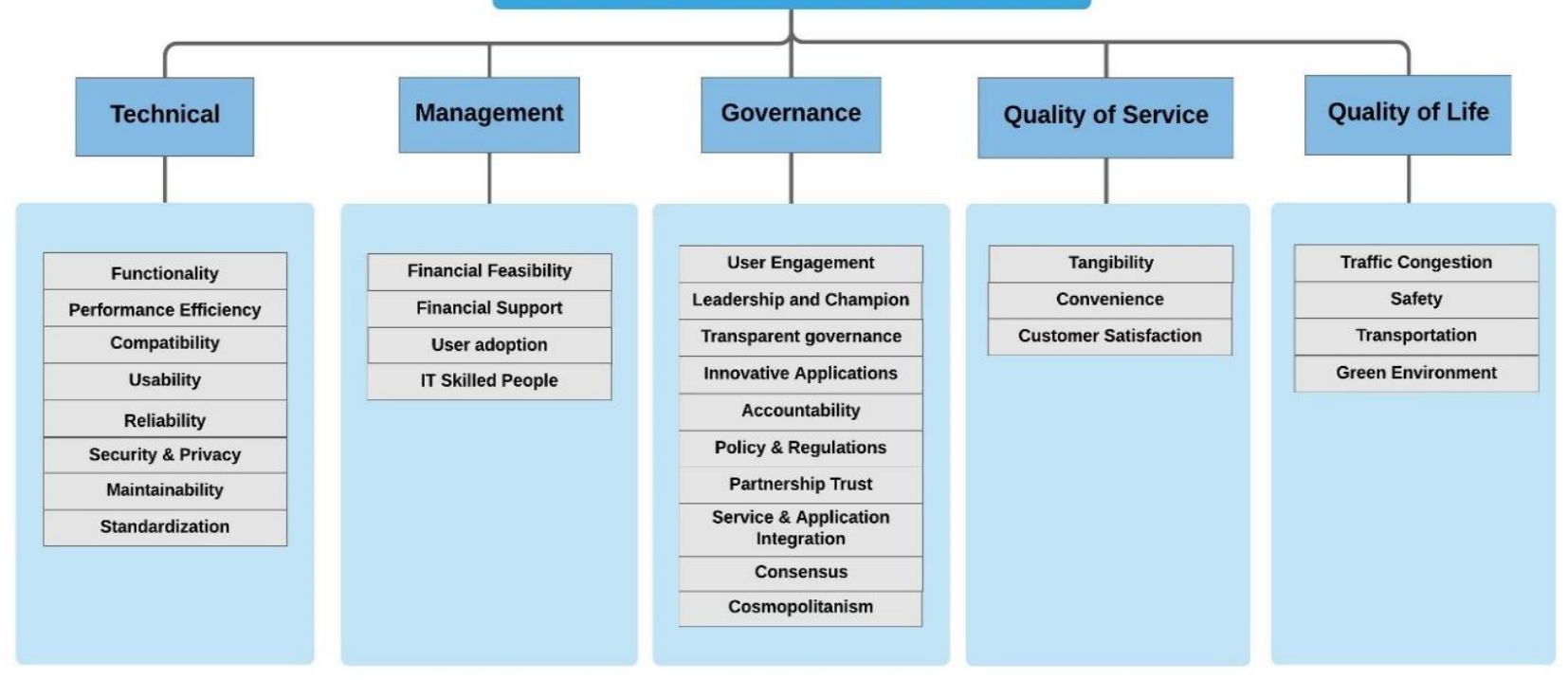

Figure 3. The Model of Critical Success Factors for Blockchain-based Intelligent Transportation Systems.

\section{4-1- Technical Context}

The CSFs classified under technical context are defined based on the software and system quality standards [25], which contains functionality, performance efficiency, compatibility, usability, reliability, security \& privacy, maintainability, and standardization, given with their subfactors in Table 1.

Table 1. Technical Context of Blockchain-based ITS Critical Success Factors.

\begin{tabular}{ccc}
\hline Critical Success Factor & Sub-Factor & Sources \\
\hline \multirow{2}{*}{ Functionality } & Functional Appropriateness & {$[25]$} \\
& Functional Completeness & {$[25]$} \\
& Functional Correctness & {$[25]$} \\
\hline \multirow{2}{*}{ Performance Efficiency } & Resource Utilization & {$[15,25]$} \\
& Time Behavior & {$[15,25]$} \\
\hline \multirow{2}{*}{ Compatibility } & Interoperability & {$[25-28]$} \\
& Co-existence & {$[25,26,29]$} \\
\hline \multirow{2}{*}{ Usability } & Accessibility & {$[15,25,30-35]$} \\
& User interface aesthetics & {$[25]$} \\
Reliability & Learnability & {$[25]$} \\
& Maturity & {$[25]$} \\
& Fault tolerance & {$[25,36,37]$} \\
& Recoverability & {$[25]$} \\
& Availability & {$[14,25]$} \\
\hline
\end{tabular}




\begin{tabular}{ccc}
\hline & Replicability & {$[25,40]$} \\
Maintainability & Stability & {$[25,40,41]$} \\
& Testability & {$[25,40]$} \\
& Scalability & {$[39,42]$} \\
\hline Standardization & - & {$[39,42,43]$} \\
\hline
\end{tabular}

\section{4-1-1- Functionality}

Functionality or functional suitability refers to a function of blockchain-based ITS to meet the stated needs under specific conditions. Functionality can be evaluated under functional appropriateness, completeness, and correctness [25]. Functional appropriateness refers to if the system or functions are appropriate for the success of the tasks and objectives [25]. Functional completeness is related to if the system covers all the specified task and user objectives [25], and functional correctness refers to if the system ensures accurate results with precision [25].

\section{4-1-2- Performance Efficiency}

The overall performance of blockchain-based ITS is an indicator, including efficiency, effectiveness, speed, and response times [15]. Performance efficiency refers to the number of resources used, and it is evaluated by time behavior and resource utilization. While time behavior indicates the response times and processing times of a system [25], resource utilization refers to the types and amounts of resources consumed in the process [25].

\section{4-1-3- Compatibility/Interoperability}

Compatibility refers to "the degree to which innovation fits with the potential adopters' existing values, previous practices, and current needs" [30]. It is considered as a significant factor for innovation success [28]. Compatibility is evaluated by co-existence and interoperability factors. While co-existence is the efficient functioning of a product or system with its environment and other systems [26], interoperability can be defined as successful interaction and communication between systems on different platforms [29].

\section{4-1-4- Usability}

Usability is defined as the satisfactory, effective, and efficient way of achieving the objectives by users [25].This CSF is similar to the factor of perceived ease of use, which is defined as "The degree of ease associated with the use of the system" [44]. The less time is required to learn and utilize the functionality of blockchain-based ITS, the more willingness to use the system exists, and the more successful the system is. It is determined as an essential CSF in the literature [30-35]. Usability is evaluated by accessibility, user interface aesthetics, and learnability. While accessibility refers to the usability of the system by a wide range of people with different characteristics and capabilities, such as color-blind people [25], user interface aesthetic covers the user satisfaction for the systems appealing design [25], and the learning factor refers to the efficient ease of use of the system [25].

\section{4-1-5- Reliability}

A reliable system performs its functions as the way they are designed for the specified time frame without failure [36,37]. Reliability is assessed by maturity, fault tolerance, recoverability, and availability [25]. Maturity indicates if the system meets the requirements of reliability under normal process conditions, while fault tolerance means proper system operation despite failures [37]. Recoverability is the ability of the system to recover damaged data after a failure and re-establishing the system on the desired state [25]. Availability refers to system readiness for use when required [25].

\section{4-1-6- Security and Privacy}

Prior studies $[6,32,33,38,39,45]$ indicated that the factors of security and privacy do not affect the success of blockchain technologies because the privacy and security through encryption is the main strength of the blockchain technologies [39]. Thus, the CSF of security and privacy covers authorization, audit, protection, and multi-location issues on the quality standards of blockchain-based ITS [25].

\section{4-1-7- Maintainability}

Maintainability of a software and information system covers scalability, replicability, testability, and stability $[25,40]$. Scalability refers to extending blockchain systems. Estimating the cost of this extension is hugely complicated [39]. The addition of new data, extending the blockchain, requires additional computational power usage for processing and validating, as well as storage for storing hashes related to the appended data. Increased blockchain size can cause inefficiency and slow transactions [42]. 
Replicability refers to successfully executing systems processes for purposed uses by a multiple of time [25]. Testability is defined as the availability of measuring the effectiveness and efficiency of the system with objective criteria [25]. Stability covers achieving expected outcomes as a result of executing processes with intended specifications [41].

\section{4-1-8- Standardization}

Required interoperability across ITS raises the need for standards and protocols. The lack of consensus protocol is a challenge for the success of blockchain-based technologies [42]. As stated in [39,43], the lack of a standard is a barrier to the success of blockchain-based ITS.

\section{4-2-Management Context}

This context covers organizational and managerial viewpoints. The success of blockchain-based ITS initiative is affected by way of performing organizational management processes of planning, organizing, directing, staffing, and controlling [46]. The CSFs defined under this context are financial feasibility, financial support, user adoption, IT skilled people, as given in Table 2 .

Table 2. Management Context of Blockchain-based ITS Success Factors.

\begin{tabular}{cc}
\hline Critical Success Factor & Sources \\
\hline Financial Feasibility & {$[10,13-15,26,47-52]$} \\
Financial Support & {$[27,33,50,53-55]$} \\
User adoption/resistance to change & {$[27,56-58]$} \\
IT Skilled People & {$[39,48,59,60]$} \\
\hline
\end{tabular}

\section{4-2-1- Financial Feasibility}

Financial Feasibility, also known as economic performance, covers productivity, profitability, revenues, and costs of blockchain-based ITS initiatives [47]. It is determined that the success factor of financial feasibility or economic performance was defined in almost all primary studies [10,13-15,26,48-52]. Blockchain-based ITS should have a feasible return on investment and short payback timeline for success, as every other innovation project. A blockchainbased ITS needs to be financially feasible and provide economic outcomes as cost efficiency and revenue to be implemented and used. Therefore, it is defined as a CSF for blockchain-based ITS.

\section{4-2-2- Financial Support}

Financial support, availability of fundings, is a significant necessity for carrying out activities in organizations [49]. Financial support is defined as an essential factor in the success of innovative technologies in the previous literature $[33,53,54]$. Having tremendous financial resources provides organizations to initiate and maintain changes and engagement in an increasingly cooperative behavior [27,55]. Budget restrictions, as a lack of resources for maintenance and operations, as well as the high implementation cost of blockchain-based ITS, make the factor of financial support as an essential CSF for blockchain-based ITS [50].

\section{4-2-3- User Adoption}

This factor is the opposite of the factor of resistance to change that is the concept of individuals or groups of people resisting changes in organizations and society because of opposing benefits [61]. Resistance to change is evaluated by routine seeking, emotional reaction, short term focus, and cognitive rigidity dimensions [61]. For example, when required financial resources are allocated promptly, a positive organizational climate is created, barriers and resistance to change are overcome more efficiently, and employees are encouraged to adopt the new system [27,56-58]. This factor was found significant for the successful implementation of innovative applications in the previous literature [27,56-58]. Thus, it is defined as a CSF for blockchain-based ITS in this study.

\section{4-2-4- IT Skilled People}

This CSF refers to the Human Resource (HR) having technical and managerial IT skills, as well as the intangible IT resources, including know-how and culture [59]. The availability of IT-skilled HR has a definite significance in the success of blockchain-based systems [39]. The factor of IT skilled People is a critical driver for success since the lack of talent pool for blockchain skills negatively affects the growth of the organization and prevents the wider adoption and usage of the blockchain technology $[48,60]$. 


\section{4-3-Governance Context}

The CSFs classified under this context are based upon improving the mechanisms for successful governance of blockchain-based ITS initiative. Governance refers to ethical decision-making and managerial actions to improve transparent governance, leadership, accountability, regulations, community involvement, etc. [62]. This context, playing a critical role in the success of blockchain-based ITS initiative, consists of user engagement, leadership and champion, transparent governance, innovative applications, service and application integration, accountability, partnership trust, community involvement, cosmopolitanism, and policy \& regulations, as given in Table 3.

Table 3. Governance Context of Blockchain-based ITS Critical Success Factors.

\begin{tabular}{cc}
\hline Critical Success Factor & Sources \\
\hline User Engagement & {$[48,63-66]$} \\
Leadership and champion & {$[48,63,64,67,68]$} \\
Transparent governance & {$[13,28,63,69-72]$} \\
Innovative Applications & {$[73,74]$} \\
Service \& application integration & {$[17,48,63,64]$} \\
Accountability & {$[63,64,75,76]$} \\
Partnership trust & {$[65,77-80]$} \\
Community Involvement & {$[19,48,81,82]$} \\
Cosmopolitanism & {$[48,83,84]$} \\
Policy \& Regulations & {$[5,39,85]$} \\
\hline
\end{tabular}

\section{4-3-1- User Engagement}

Participative and collaborative user engagement for digital technologies can work as a catalyst for providing better services and products for broader opportunities [66]. The rise of smart city initiatives causes gaining user engagement more significant for engaging users in the governance and management design and procedures. As the level of engagement increases, users could positively influence the projects from their perspectives [64]. Thus, the value realized, the motivation to use, and the usability level for the innovative product or service also increases $[48,65]$. Therefore, user engagement is defined as a CSF for blockchain-based ITS initiatives.

\section{4-3-2- Leadership and Champion}

Executive leadership needs to understand the innovative technology to create relevant use-cases for the project. Blockchain integrated systems offer a new way of leadership, which is based upon decentralized authority. Although it is believed that centralized management is a better way to create standardization, efficiency, and profitability [48,68], the blockchain technology provides decentralization on management and power diffusion within the organization to improve the success of the projects. Therefore, leadership is a vital CSF on blockchain-based ITS.

\section{4-3-3- Transparent Governance}

Transparent governance refers to open communication and disclosure of information between citizens and organizations or governments by considering privacy constraints [71,72]. Blockchain technology provides a secure and transparent transaction for application, which ensures the non-tampering of any information. Also, the decentralized authority and the ledger system distribution between all parties enable transparency [13,28]. Increasing transparency, providing users to be able to track all functions of the systems, contributes to increasing trust towards the organization and applications [86]. Correspondingly, it affects the success of blockchain-based ITS initiatives in a positive manner. Thus, transparent governance is defined as a CSF for blockchain-based ITS.

\section{4-3-4- Innovative Applications}

The number of successful new innovative solutions developed for complementing the product or service produced by emergent technologies is a crucial factor in measuring the success of emergent technologies. The innovative applications that are generated from several resources, such as open government data or user-generated data, provide economic growth by producing new products or services by startups [74]. From the perspective of transportation systems, city governments have adopted many new solutions and are still seeking new ways of improvements with new technologies [73]. With the goal of economic growth, the factor of innovative applications is a vital CSF for the success of blockchain-based ITS. 


\section{4-3-5- Service and Application Integration}

One of the benefits of blockchain technologies is the capability of integration with other systems and applications that are developed based on different technologies since it has a structure of open independent P2P network without a central authority [48]. However, there are also some challenges encountered in the integration of blockchain into ITS, as security \& privacy, performance, incentive mechanisms, and optimized consensus [17]. The more integration with other systems in real-time can be provided, the more functionality and the more usage of the system are obtained. Therefore, service and application integration is considered as a critical CSF for blockchain-based ITS.

\section{4-3-6- Accountability}

Accountability can be defined as "the right of some actors to hold other actors to a set of standards, to judge whether they have fulfilled their responsibilities in light of these standards, and to impose sanctions if they determine that these responsibilities have not been met" [75]. Blockchain technology, with its distributed architecture, decentralized authority, cryptography protocols, and consensus algorithms, facilitate transparency and accountability, which provides trust among participants [76]. Thus, increased accountability on the governance process is an essential CSF for blockchain-based ITS.

\section{4-3-7- Partnership Trust}

This CSF can be defined as "the willingness of a party to be vulnerable to the actions of another party based on the expectation that the other will perform a particular action important to the trustor, irrespective of the ability to monitor or control that other party" [79]. As indicated by prior studies in the literature, inter-organizational trust affects the successful adoption of the new technology [77,78]. The previous literature $[65,80]$ showed the significance of inter-organizational trust on the successful adoption of blockchain technologies.

\section{4-3-8- Consensus/Community Involvement/Engaged Communities}

In order to achieve success in the transportation domain, achieving the consensus of the involved parties of citizens, transportation employees, government, municipalities, civil society organizations, and communities of technology providers plays a critical role. The success of blockchain technologies is based upon having a strong community that establishes a value-creating network. The technology choices and regulations shared solutions must be established by the collaboration of industry leaders for a successful result. Collaboration on solutions and regulations regarding technology choices are required by the industry leaders [87]. The knowledge should be shared with competitors for faster development on infrastructure and generation of new projects. One example of community involvement in blockchain-based ITS applications is voting on the signal time of traffic lights [19]. There have been several collaborations on financial and other industries as R3CEV, the Hyperledger Project, the Post Trade Distributed Ledger Group, Blockchain Insurance Industry Initiative (B3i), Healthcare Blockchain Consortium, and Commodity Blockchain Consortium [48]. This makes community involvement as a vital CSF for blockchain-based ITS.

\section{4-3-9- Cosmopolitanism}

Cosmopolitanism is defined with greater world openness, global awareness, loyalty to humanity, self-reflection, and self-problematization to engaging new communities [84]. With emerging technologies, citizens have higher interaction opportunities for collaboration and participation in the governance of projects. The governance of blockchain-based ITS requires open-mindedness for the development and execution of new ways of transportation practices with the inclusion of every community and collaboration partner [48]. Thus, cosmopolitanism is defined as a substantial CSF for blockchain-based ITS.

\section{4-3-10- Policy \& Regulations}

This CSF is defined as the policy and regulations provided by the government to regulate and monitor the industries for the usage of new technology. It is found as a fundamental factor affecting innovation diffusion [85]. Since blockchain is a newly developed technology and preparation of new regulation includes some challenges, such as the definition of ownership of digital records and access rights, the law and regulations have not been established yet [5]. This can encourage or discourage adoption, correspondingly the success of blockchain-based ITS projects. There is an urgent need to develop and manage such standards [39]. Thus, the CSF of policy \& regulations is defined to measure the success of the blockchain-based ITS project.

\section{4-4-Quality of Service Context}

The CSFs considered under this context are based on the service quality perceived and expected by society. Quality of service is defined as "the degree and discrepancy between customers' perceptions and expectations" [88]. Since 
transportation systems affect every individual, the expected and perceived quality of service is a relevant context. The quality of service context of the proposed model consists of tangibility, convenience, and customer satisfaction, as given in Table 4.

Table 4. Quality of Service Context of Blockchain-based ITS Critical Success Factors.

\begin{tabular}{cc}
\hline Critical Success Factor & Sources \\
\hline Tangibility & {$[88,89]$} \\
Convenience & {$[90-92]$} \\
Customer Satisfaction & {$[75]$} \\
\hline
\end{tabular}

\section{4-1-1- Tangibility}

Tangibility refers to the physical features of the services, including appearance, tools and equipment, personnel, communication materials, comfort, cleanness, other features used to provide the service and other customers in the establishment $[88,89]$. Tangible factors on transportation systems are generally comfort provided and cleanness of the transportation system. Tangibility is an essential factor for the customers' usage of blockchain-based ITS. Thus, it is defined as a CSF for blockchain-based ITS.

\section{4-1-2- Convenience}

Convenience refers to customers' time and effort perceptions about buying or using a service [90]. Convenience is one of the goals of ITS as providing accessible service to travelers [91]. In the context of ITS, convenient ticketing, and convenient durations of waiting and transportation are some examples of convenience. The higher convenience of the service provided, the higher the success of blockchain-based ITS are achieved.

\section{4-1-3- Customer Satisfaction}

Customer satisfaction can be achieved by delivering high customer value with products and services [75]. Blockchain transparency can remove intermediaries, which results in lower prices and provide useful and verifiable information on a product or service. Also, the authenticity of records on the blockchain provides a sense of security for the customers participating [75]. As defined in the previous literature, customer satisfaction for the service provided indicates a signal for the project success [93]. Since customers are a vital part of the system, customer satisfaction is defined as a CSF for blockchain-based ITS.

\section{4-5- Quality of Life Context}

Quality of life is used for the assessment of individuals and societies of overall well-being [94]. "ITS applies advanced communication, information, and electronics technology to solve the global transportation problems such as congestion, transport efficiency, safety, and environmental concerns" [95]. One of the expected outcomes of ITS are the improvement of the quality of life for society by providing cleaner, safer, and convenient transportation [96]. Quality of Life context of the proposed model consists of CSFs of traffic congestion, safety, and transportation and green environment, as given in Table 5.

Table 5. Quality of Life Context of Blockchain-based ITS Success Factors.

\begin{tabular}{cc}
\hline Critical Success Factor & Sources \\
\hline Traffic Congestion & {$[73,95]$} \\
Safety & {$[73,95]$} \\
Transportation & {$[73,95]$} \\
Green Environment & {$[73]$} \\
\hline
\end{tabular}

\section{4-5-1- Traffic Congestion}

Congestion is a global phenomenon that urban cities are facing. The increasing population on cities and infrastructure on suburban areas development speed lagging causes daily travel time of people to increase and created congestions within the city [73]. Advanced Traffic Management Systems (ATMS) and Advanced Transport Pricing Systems (ATPS) are examples of congestion management. One of the main goals of ITS projects are to reduce traffic congestion, which results in the success of the system. There is a positive correlation between the rate of decreasing traffic congestion and the success of the blockchain-based ITS initiative. Thus, Traffic congestion is defined as a vital CSF on blockchain-based ITS. 


\section{4-5-2- Safety}

Safety in the context of transportation systems refers to road and vehicle safety [73]. Measurement of safety refers to the number of accidents. One of the aims of emerging ITS projects are to reduce the number of human-caused accidents by utilization of emerging technologies. Maintenance and Construction Management (MCM) is an example of road safety which maintains roadways, manage construction, and clear snow [73]. Another is Emergency Management (EM), which provides emergency medical serves and information on disasters. The fewer accidents and safer transportation are observed, the more successful blockchain-based ITS are achieved. Thus, safety is defined as a CSF for blockchain-based ITS.

\section{4-5-3- Transportation}

This CSF covers the time spent during active and non-active traveling. While the worlds' population increases, cities are faced with increased traffic congestions, which results in a large amount of time spent on transportation. ITS has a goal to reduce this time, correspondingly to increase the quality of life of citizens. Examples of ITS related to reducing time spent during transportation are Advanced Public Transportation Systems (APTS), which offer safe, reliable, and efficient public transportation, and Advanced Travellers Information Systems (ATIS), which provides travel data to commuters $[73,95]$. The less time spent on transportation is provided, the more successful blockchainbased ITS is. Thus, it is defined as a CSF to measure the success of blockchain-based ITS.

\section{4-5-4- Green Environment}

The green environment, also known as environmental conservation, for the context of transportation systems is regarding the energy efficiency and reduction of carbon dioxide emissions to provide a cleaner environment [73]. Examples of ITS on the green environment are ATIS, informing users about efficient travel routes for minimum energy consumption, and APTS, providing public transportation by reducing the number of cars on traffic to helps to reduce carbon dioxide emissions. It can be asserted that the blockchain-based ITS initiative, providing less carbon dioxide emission, is a more successful initiative. Thus, the CSF of the green environment is stated in the proposed model.

\section{5- Conclusion}

As one of the most disruptive emerging technologies, blockchain is transforming all industries by disrupting existing business operations at an unprecedented pace. Although it offers new opportunities by providing improvement in transparency, immutability, traceability, and efficiency for the ITS, it has not yet been fully adopted. It is still at the infancy stage. This study suggests critical insights that may be utilized by ITS providers to design their products, and by industry leaders to increase the success of their blockchain-based ITS projects. A model of CSFs is developed by identification of the main contexts and related CSFs and sub-factors (if there is), to provide successful implementation of blockchain-based ITS. The developed model of CSFs for blockchain-based ITS consist of five main contexts of technical, governance, management, quality of life, and quality of service and 29 CSFs defined under these contexts. The eight CSFs defined under technical context are functionality, performance efficiency, compatibility, usability, reliability, security \& privacy, maintainability, and standardization. The four CSFs defined under the context of management are financial feasibility, financial support, user adoption, and IT skilled people. The context of governance consists of ten CSFs of user engagement, leadership, and champion, transparent governance, innovative applications, service \& application integration, accountability, partnership trust, community involvement, cosmopolitanism, and policy \& regulations. Lastly, the context of Quality of Life consists of four CSFs of traffic congestion, safety, and transportation, and a green environment.

As a result of SLR, it was observed that there is not any study investigating CSFs for blockchain-based ITS in the literature. One of the main contributions of this study is to fill this gap in the literature by proposing a comprehensive model of CSFs for blockchain-based ITS by capturing the viewpoints of different stakeholders. The second contribution of this study is to create a foundation and shed light on the directions for future research and development.

As future work, it is planned to identify the Key Performance Indicators (KPIs) for each CSF and sub-factor as well as to conduct a case study for validating the usefulness and adequacy of the proposed model.

\section{6- Conflict of Interest}

The author declares that there is no conflict of interests regarding the publication of this manuscript. In addition, the ethical issues, including plagiarism, informed consent, misconduct, data fabrication and/or falsification, double publication and/or submission, and redundancies have been completely observed by the authors. 


\section{7- References}

[1] Khazraeian, Samaneh, and Mohammed Hadi. "Intelligent Transportation Systems in Future Smart Cities." In Studies in Systems, Decision and Control, 186:109-20. Springer, 2019. doi:10.1007/978-3-319-98923-5_6.

[2] Union, International Telecommunication. Intelligent Transport Systems, 2007.

[3] Joseph, A.D., A.R. Beresford, J. Bacon, D.N. Cottingham, J.J. Davies, B.D. Jones, Haitao Guo, et al. "Intelligent Transportation Systems.” IEEE Pervasive Computing 5, no. 4 (October 2006): 63-67. doi:10.1109/mprv.2006.77.

[4] Sumalee, Agachai, and Hung Wai Ho. "Smarter and More Connected: Future Intelligent Transportation System." IATSS Research 42, no. 2 (2018): 67-71. doi:10.1016/j.iatssr.2018.05.005.

[5] Gökalp, Ebru, Mert Onuralp Gökalp, Selin Çoban, and P Erhan Eren. "Analysing Opportunities and Challenges of Integrated Blockchain Technologies in Healthcare.” In Lecture Notes in Business Information Processing, 333:174-83. Springer, 2018. doi:10.1007/978-3-030-00060-8_13.

[6] Swan, Melanie. Blockchain: Blueprint for a new economy. " O'Reilly Media, Inc.", 2015

[7] Pawczuk, Linda, Rob Massey, and Jonathan Holdowsky. "Deloitte' s 2019 Global Blockchain Survey - Blockchain Gets down to Business." Deloitte Insights, 2019. https://www2.deloitte.com/content/dam/insights/us/articles/2019-global-blockchainsurvey/DI_2019-global-blockchain-survey.pdf.

[8] Leidecker, Joel K., and Albert V. Bruno. "Identifying and Using Critical Success Factors.” Long Range Planning 17, no. 1 (1984): 23-32. doi:10.1016/0024-6301(84)90163-8.

[9] Sussman, Joseph S. Perspectives on Intelligent Transportation Systems (ITS). Springer Science \& Business Media, 2008.

[10] Yang, Mei Hsiang, Tzong Ru Lee, and Tin Chang Chang. "Key Success Factors of Blockchain Platform for Micro-Enterprises." Journal of Asian Finance, Economics and Business 6, no. 3 (2019): 283-93. doi:10.13106/jafeb.2019.vol6.no3.283.

[11] Yuan, Yong, and Fei-Yue Wang. “Towards Blockchain-Based Intelligent Transportation Systems.” 2016 IEEE 19th International Conference on Intelligent Transportation Systems (ITSC) (November 2016). doi:10.1109/itsc.2016.7795984.

[12] Crosby, Michael, Pradan Pattanayak, Sanjeev Verma, and Vignesh Kalyanaraman. "Blockchain Technology: Beyond Bitcoin." Applied Innovation 2, no. 6-10 (2016): 71.

[13] Fernando, Erick, Meyliana, and Surjandy. "Success Factor of Implementation Blockchain Technology in Pharmaceutical Industry: A Literature Review.” In 2019 6th International Conference on Information Technology, Computer and Electrical Engineering, ICITACEE 2019, 1-5. IEEE, 2019. doi:10.1109/ICITACEE.2019.8904335.

[14] Surjandy, Erick Fernando, Meyliana, Raymond Kosala, Harco Leslie Hendric Spits Warnars, Edi Abdurachman, and Suhono Harso Supangkat. "Success Factors of the Blockchain Adoption for Smart Manufacture." In 2018 International Seminar on Research of Information Technology and Intelligent Systems, ISRITI 2018, 2018-Janua:617-21. IEEE, 2018. doi:10.1109/ISRITI.2018.8864253

[15] Yadav, Sachin, and Surya Prakash Singh. "Blockchain Critical Success Factors for Sustainable Supply Chain." Resources, Conservation and Recycling 152 (2020): 104505. doi:10.1016/j.resconrec.2019.104505.

[16] La'Zooz. Available Online: http://lazooz.org (Accessed on 26 July 2020).

[17] Mollah, Muhammad Baqer, Jun Zhao, Dusit Niyato, Yong Liang Guan, Chau Yuen, Sumei Sun, Kwok-Yan Lam, and Leong Hai Koh. "Blockchain for the Internet of Vehicles towards Intelligent Transportation Systems: A Survey." ArXiv E-Prints, 2020, arXiv-2007.

[18] Lopez, David, and Bilal Farooq. "A Blockchain Framework for Smart Mobility.” In 2018 IEEE International Smart Cities Conference, ISC2 2018, 1-7. IEEE, 2019. doi:10.1109/ISC2.2018.8656927.

[19] Cheng, Lichen, Jiqiang Liu, Guangquan Xu, Zonghua Zhang, Hao Wang, Hong Ning Dai, Yulei Wu, and Wei Wang. "SCTSC: A Semicentralized Traffic Signal Control Mode with Attribute-Based Blockchain in IoVs." IEEE Transactions on Computational Social Systems 6, no. 6 (2019): 1373-85. doi:10.1109/TCSS.2019.2904633.

[20] Oaken Innovative. Available Online: https://www.oakeninnovations.com (accessed on 25 July 2020).

[21] Leiding, Benjamin, and Wiliam V. Vorobev. "Enabling the V2X Economy Revolution Using a Blockchain-Based Value Transaction Layer for Vehicular Ad-Hoc Networks.” In Conference on Information Systems (MCIS), 1-31, 2018.

[22] Leiding, Benjamin, Parisa Memarmoshrefi, and Dieter Hogrefe. "Self-Managed and Blockchain-Based Vehicular Ad-Hoc Networks." Proceedings of the 2016 ACM International Joint Conference on Pervasive and Ubiquitous Computing: Adjunct (September 12, 2016). doi:10.1145/2968219.2971409.

[23] Singh, Madhusudan, and Shiho Kim. "Intelligent Vehicle-Trust Point: Reward Based Intelligent Vehicle Communication Using Blockchain.” ArXiv Preprint ArXiv:1707.07442, 2017. 
[24] Kitchenham, Barbara. "Procedures for Performing Systematic Reviews.” Keele, UK, Keele University 33, no. TR/SE-0401 (2004): 28. doi:10.1.1.122.3308.

[25] Publication, B S I Standards. "BSI Standards Publication Systems and Software Engineering — Systems and Software Quality Requirements and Evaluation ( SQuaRE ) — Measurement of System and Software Product Quality,” 2016.

[26] Hussein, W. N., L. M. Kamarudin, H. N. Hussain, N. A. Ishak, A. Zakaria, and K. J. Jadaa. "Discovering the Implementation Success Factors for IoT and Big Data Analytics in Transportation System.” In IOP Conference Series: Materials Science and Engineering, 705:12049. IOP Publishing, 2019. doi:10.1088/1757-899X/705/1/012049.

[27] Wang, Yu Min, Yi Shun Wang, and Yong Fu Yang. "Understanding the Determinants of RFID Adoption in the Manufacturing Industry.” Technological Forecasting and Social Change 77, no. 5 (2010): 803-15. doi:10.1016/j.techfore.2010.03.006.

[28] Park, Seoyeon, Jiyun Kim, Dukshin Oh, and Jongwan Kim. "Evaluation of Blockchain Business Success Factors Using Ahp." Indian Journal of Computer Science and Engineering 11, no. 2 (2020): 99-111. doi:10.21817/indjcse/2020/v11i2/201102013.

[29] Rogers Everett, M. “Diffusion of Innovations.” New York, 1995.

[30] Francisco, Kristoffer, and David Swanson. "The Supply Chain Has No Clothes: Technology Adoption of Blockchain for Supply Chain Transparency.” Logistics 2, no. 1 (2018): 2. doi:10.3390/logistics2010002.

[31] Kamble, Sachin, Angappa Gunasekaran, and Himanshu Arha. "Understanding the Blockchain Technology Adoption in Supply Chains-Indian Context." International Journal of Production Research 57, no. 7 (2019): $2009-33$. doi:10.1080/00207543.2018.1518610.

[32] Yusof, Hayati, Mai Farhana Mior Badrul Munir, Zulnurhaini Zolkaply, Chin Li Jing, Chooi Yu Hao, Ding Swee Ying, Lee Seang Zheng, Ling Yuh Seng, and Tan Kok Leong. "Behavioral Intention to Adopt Blockchain Technology: Viewpoint of the Banking Institutions in Malaysia.” International Journal of Advanced Scientific Research and Management 3 (2018).

[33] Chana, Felix T.S., and Alain Yee Loong Chong. "Determinants of Mobile Supply Chain Management System Diffusion: A Structural Equation Analysis of Manufacturing Firms." International Journal of Production Research 51, no. 4 (2013): 11961213. doi:10.1080/00207543.2012.693961.

[34] Low, Chinyao, Yahsueh Chen, and Mingchang Wu. "Understanding the Determinants of Cloud Computing Adoption." Industrial Management and Data Systems 111, no. 7 (2011): 1006-23. doi:10.1108/02635571111161262.

[35] Premkumar, G, Keshavamurthy Ramamurthy, and Sree Nilakanta. "Implementation of Electronic Data Interchange: An Innovation Diffusion Perspective.” Journal of Management Information Systems 11, no. 2 (1994): 157-86.

[36] Şener, Umut, Ebru Gökalp, and P. Erhan Eren. "Cloud-Based Enterprise Information Systems: Determinants of Adoption in the Context of Organizations." In Communications in Computer and Information Science, edited by Giedre DregvaitRobertas Damasevicius, 639:53-66, 2016. doi:10.1007/978-3-319-46254-7_5.

[37] Ahmed, Waseem, and Yong Wei Wu. “A Survey on Reliability in Distributed Systems.” Journal of Computer and System Sciences 79, no. 8 (2013): 1243-55. doi:10.1016/j.jcss.2013.02.006.

[38] Angelis, Jannis, and Elias Ribeiro da Silva. "Blockchain Adoption: A Value Driver Perspective.” Business Horizons 62, no. 3 (2019): 307-14. doi:10.1016/j.bushor.2018.12.001.

[39] Wang, Huaiqing, Kun Chen, and Dongming Xu. “A Maturity Model for Blockchain Adoption.” Financial Innovation 2, no. 1 (December 2016): 12. doi:10.1186/s40854-016-0031-z.

[40] Garba, Salisu, Babangida Isyaku, and Mujahid Abdullahi. "Data-Driven Model for Non-Functional Requirements in Mobile Application Development." International Journal of Computer Science and Information Technology 11, no. 02 (2019): 97-109. doi:10.5121/ijcsit.2019.11209.

[41] EL-Khalil, Raed, Zachary Moran Leffakis, and Paul C. Hong. "Impact of Improvement Tools on Standardization and Stability Goal Practices : An Empirical Examination of US Automotive Firms.” Journal of Manufacturing Technology Management 31, no. 4 (2020): 705-23. doi:10.1108/JMTM-08-2019-0289.

[42] Makhdoom, Imran, Mehran Abolhasan, Haider Abbas, and Wei Ni. "Blockchain's Adoption in IoT: The Challenges, and a Way Forward." Journal of Network and Computer Applications. Elsevier, 2019. doi:10.1016/j.jnca.2018.10.019.

[43] Holotiuk, Friedrich, and Jürgen Moormann. "Organizational Adoption of Digital Innovation: The Case of Blockchain Technology.” In 26th European Conference on Information Systems: Beyond Digitization - Facets of Socio-Technical Change, ECIS 2018, 2018.

[44] Venkatesh, Viswanath, Michael G. Morris, Gordon B. Davis, and Fred D. Davis. "User Acceptance of Information Technology: Toward a Unified View.” MIS Quarterly: Management Information Systems 27, no. 3 (2003): 425-78. doi:10.2307/30036540.

[45] Gökalp, Ebru, Mert Onuralp Gökalp, and Selin Çoban. "Blockchain-Based Supply Chain Management: Understanding the Determinants of Adoption in the Context of Organizations." Information Systems Management, September 12, $2020,1-22$. doi:10.1080/10580530.2020.1812014. 
[46] Ni, Xiaochun, Shuai Zeng, Xuan Han, Yong Yuan, and Fei-Yue Wang. "Organizational Management Using Software-Defined Robots Based on Smart Contracts.” 2018 IEEE Intelligent Vehicles Symposium (IV) (June 2018). doi:10.1109/ivs.2018.8500708.

[47] Belvedere, Valeria, and Alberto Grando. "ICT-Enabled Time Performance: An Investigation of Value Creation Mechanisms." Production Planning \& Control (September 20, 2016): 1-14. doi:10.1080/09537287.2016.1233359.

[48] Prasad, Sanjay, Ravi Shankar, Rachita Gupta, and Sreejit Roy. “A TISM Modeling of Critical Success Factors of Blockchain Based Cloud Services.” Journal of Advances in Management Research 15, no. 4 (2018): 434-56. doi:10.1108/JAMR-03-20180027.

[49] Nayak, Gurudutt, and Amol S. Dhaigude. "A Conceptual Model of Sustainable Supply Chain Management in Small and Medium Enterprises Using Blockchain Technology.” Cogent Economics and Finance 7, no. 1 (2019): 1667184. doi:10.1080/23322039.2019.1667184.

[50] Mokaddem, Youssef El, Fouad Jawab, and Lissane Elhaq Saad. "Intelligent Transportations Systems : Review of Current Challenges and Success Factors: The Case of Developing Countries." In International Colloquium on Logistics and Supply Chain Management, LOGISTIQUA 2019, 1-6. IEEE, 2019. doi:10.1109/LOGISTIQUA.2019.8907308

[51] Hastig, Gabriella M., and Man Mohan S. Sodhi. "Blockchain for Supply Chain Traceability: Business Requirements and Critical Success Factors.” Production and Operations Management 29, no. 4 (2020): 935-54. doi:10.1111/poms.13147.

[52] Surjandy, Erick Fernando, and Meyliana. "Essential Blockchain Technology Adoption Factors in Pharmaceutical Industry.” In 2019 4th International Conference on Information Technology, Information Systems and Electrical Engineering, ICITISEE 2019, 523-26. IEEE, 2019. doi:10.1109/ICITISEE48480.2019.9003997.

[53] Chong, Alain Yee Loong, and Felix T.S. Chan. "Structural Equation Modeling for Multi-Stage Analysis on Radio Frequency Identification (RFID) Diffusion in the Health Care Industry.” Expert Systems with Applications 39, no. 10 (August 2012): 8645-54. doi:10.1016/j.eswa.2012.01.201.

[54] Oliveira, Tiago, and Maria Fraga Martins. "Information Technology Adoption Models at Firm Level: Review of Literature.” In 4th European Conference on Information Management and Evaluation, ECIME 2010, 14:312-22. Academic Conferences International Limited, 2010.

[55] Weiner, Bryan J. “A Theory of Organizational Readiness for Change.” Implementation Science 4, no. 1 (2009): 67. doi:10.1186/1748-5908-4-67.

[56] Gangwar, Hemlata, Hema Date, and R. Ramaswamy. "Understanding Determinants of Cloud Computing Adoption Using an Integrated TAM-TOE Model.” Journal of Enterprise Information Management 28, no. 1 (February 9, 2015): 107-30. doi:10.1108/JEIM-08-2013-0065.

[57] Dong, Linying, Derrick Neufeld, and Chris Higgins. "Top Management Support of Enterprise Systems Implementations." Journal of Information Technology 24, no. 1 (March 2009): 55-80. doi:10.1057/jit.2008.21.

[58] Sabherwal, Rajiv, Anand Jeyaraj, and Charles Chowa. "Information System Success: Individual and Organizational Determinants.” Management Science 52, no. 12 (December 2006): 1849-64. doi:10.1287/mnsc.1060.0583.

[59] Grant, Robert M. "The Resource-Based Theory of Competitive Advantage: Implications for Strategy Formulation.” California Management Review 33, no. 3 (April 1991): 114-35. doi:10.2307/41166664.

[60] Castillo, Michael del. "The Lack of Blockchain Talent Is Becoming An Industry Concern,” 2017. https://www.coindesk.com/blockchain-hiring-difficulties-becoming-industry-concern/.

[61] Oreg, Shaul. "Resistance to Change: Developing an Individual Differences Measure.” Journal of Applied Psychology 88, no. 4 (2003): 680-93. doi:10.1037/0021-9010.88.4.680.

[62] Muller, Ralf. "Project Governance.” Strategic Direction, 2011.

[63] Joshi, Sujata, Saksham Saxena, Tanvi Godbole, and Shreya. "Developing Smart Cities: An Integrated Framework." Procedia Computer Science 93 (2016): 902-9. doi:10.1016/j.procs.2016.07.258.

[64] Chourabi, Hafedh, Taewoo Nam, Shawn Walker, J. Ramon Gil-Garcia, Sehl Mellouli, Karine Nahon, Theresa A. Pardo, and Hans Jochen Scholl. "Understanding Smart Cities: An Integrative Framework." In Proceedings of the Annual Hawaii International Conference on System Sciences, 2289-97. IEEE, 2012. doi:10.1109/HICSS.2012.615.

[65] Queiroz, Maciel M., and Samuel Fosso Wamba. "Blockchain Adoption Challenges in Supply Chain: An Empirical Investigation of the Main Drivers in India and the USA." International Journal of Information Management 46, no. 1 (June 2019): 70-82. doi:10.1016/j.ijinfomgt.2018.11.021

[66] Townsend, Anthony M. Smart Cities: Big Data, Civic Hackers, and the Quest for a New Utopia. WW Norton \& Company, 2013. 
[67] Dearing, Tiziana C, and Mary J Cronin. Managing for Social Impact. [Electronic Resource] : Innovations in Responsible Enterprise. Springer EBooks. Springer, 2017.

[68] Alkış, Nurcan, Murat Tahir Çaldağ, and Ebru Gökalp. “An Integrated Holistic Success Model for Evaluating Smart City Initiatives." In Proceedings on the International Conference on Internet Computing (ICOMP), 16-21. The Steering Committee of The World Congress in Computer Science, Computer ..., 2019.

[69] Caird, Sally P., and Stephen H. Hallett. “Towards Evaluation Design for Smart City Development.” Journal of Urban Design, 2018, 1-22. doi:10.1080/13574809.2018.1469402.

[70] Monzon, A. "Smart Cities Concept and Challenges: Bases for the Assessment of Smart City Projects. University of Madrid Transport Research Centre.” In 2015 International Conference on Smart Cities and Green ICT Systems (SMARTGREENS), 1-11. IEEE, 2015.

[71] Çaldağ, Murat Tahir, Ebru Gökalp, and Nurcan Alkış. "Analyzing Determinants of Open Government Based Technologies and Applications Adoption in the Context of Organizations." In Proceedings of the International Conference on E-Learning, eBusiness, Enterprise Information Systems, and e-Government (EEE), 50-56. The Steering Committee of The World Congress in Computer Science, Computer ..., 2019.

[72] Abu-Shanab, Emad A. "Reengineering the Open Government Concept: An Empirical Support for a Proposed Model." Government Information Quarterly 32, no. 4 (2015): 453-63. doi:10.1016/j.giq.2015.07.002.

[73] Frost, A, and Sullivan White Paper. "A Guide to INTELLIGENT TRANSPORTATION SYSTEMS \& BEST PRACTISES," (2018).

[74] Çaldă̆, Murat Tahir, Mert Onuralp Gökalp, and Ebru Gökalp. "Open Government Data: Analysing Benefits and Challenges.” In 2019 1st International Informatics and Software Engineering Conference (UBMYK), 1-6. IEEE, 2019.

[75] Kshetri, Nir. "Blockchain and the Economics of Customer Satisfaction.” IT Professional 21, no. 1 (2019): $93-97$.

[76] Rizal Batubara, F, Jolien Ubacht, and Marijn Janssen. “Unraveling Transparency and Accountability in Blockchain.” In Proceedings of the 20th Annual International Conference on Digital Government Research, 204-13, 2019.

[77] Wu, Kewen, Yuxiang Zhao, Qinghua Zhu, Xiaojie Tan, and Hua Zheng. "A Meta-Analysis of the Impact of Trust on Technology Acceptance Model: Investigation of Moderating Influence of Subject and Context Type.” International Journal of Information Management 31, no. 6 (2011): 572-81. doi:10.1016/j.jinfomgt.2011.03.004c.

[78] Lin, Hsiu Fen. "An Empirical Investigation of Mobile Banking Adoption: The Effect of Innovation Attributes and KnowledgeBased Trust." International Journal of Information Management 31, no. 3 (June 2011): 252-60. doi:10.1016/j.ijinfomgt.2010.07.006.

[79] Schoorman, F. David, Roger C. Mayer, and James H. Davis. “An Integrative Model of Organizational Trust: Past, Present, and Future.” Academy of Management Review 32, no. 2 (April 2007): 344-54. doi:10.5465/amr.2007.24348410.

[80] Sharma, Mahak, Ruchita Gupta, and Padmanav Acharya. "Prioritizing the Critical Factors of Cloud Computing Adoption Using Multi-Criteria Decision-Making Techniques." Global Business Review 21, no. 1 (February 23, 2020): $142-61$. doi:10.1177/0972150917741187.

[81] Cavoukian, Ann, and Michelle Chibba. "Cognitive Cities, Big Data and Citizen Participation: The Essentials of Privacy and Security.” In Studies in Systems, Decision and Control, 63:61-82. Springer, 2016. doi:10.1007/978-3-319-33798-2_4.

[82] Zheng, Zibin, Shaoan Xie, Hongning Dai, Xiangping Chen, and Huaimin Wang. "An Overview of Blockchain Technology: Architecture, Consensus, and Future Trends.” In 2017 IEEE International Congress on Big Data (BigData Congress), 557-64. IEEE, 2017

[83] Benamrou, Badr, Benahmed Mohamed, Abdes Samed Bernoussi, and Ouardouz Mustapha. "Ranking Models of Smart Cities." In Colloquium in Information Science and Technology, CIST, 872-79. IEEE, 2017. doi:10.1109/CIST.2016.7805011.

[84] Hollinger, David A. "Not Universalists, Not Pluralists: The New Cosmopolitans Find Their Own Way." In Conceiving Cosmopolitanism: Theory, Context, and Practice, 227-39. Oxford: Oxford UP, 2002.

[85] Zhu, Kevin, Kenneth L. Kraemer, and Sean Xu. "The Process of Innovation Assimilation by Firms in Different Countries: A Technology Diffusion Perspective on e-Business.” Management Science 52, no. 10 (2006): 1557-76. doi:10.1287/mnsc.1050.0487.

[86] Mayer, Roger C, James H Davis, and F David Schoorman. “An Integrative Model of Organizational Trust.” Academy of Management Review 20, no. 3 (1995): 709-34.

[87] Buehler, Kevin, Daniele Chiarella, Helmut Heidegger, Matthieu Kemerle, Akash Lai, and Jared Moon. "Beyond the Hype: Blockchains in Capital Markets.” McKinsey Working Papers on Corporate \& Investment Banking, no. 12 (2015): 32. doi:10.1016/S0034-9356(11)70061-5. 
[88] Parasuraman, Ananthanarayanan, Valarie A Zeithaml, and Leonard L Berry. "Servqual: A Multiple-Item Scale for Measuring Consumer Perc." Journal of Retailing 64, no. 1 (1988): 12.

[89] Santos, Jessica. "From Intangibility to Tangibility on Service Quality Perceptions: A Comparison Study between Consumers and Service Providers in Four Service Industries.” Managing Service Quality: An International Journal, 2002.

[90] Berry, Leonard L, Kathleen Seiders, and Dhruv Grewal. “Understanding Service Convenience.” Journal of Marketing 66, no. 3 (2002): 1-17.

[91] Lin, Yangxin, Ping Wang, and Meng Ma. "Intelligent Transportation System (Its): Concept, Challenge and Opportunity.” In 2017 Ieee 3rd International Conference on Big Data Security on Cloud (Bigdatasecurity), Ieee International Conference on High Performance and Smart Computing (Hpsc), and Ieee International Conference on Intelligent Data and Security (Ids), 167-72. IEEE, 2017.

[92] Diakaki, Christina, Markos Papageorgiou, Ioannis Papamichail, and Ioannis Nikolos. "Overview and Analysis of Vehicle Automation and Communication Systems from a Motorway Traffic Management Perspective." Transportation Research Part A: Policy and Practice 75 (2015): 147-65.

[93] Narayanan, Sriram, Sridhar Balasubramanian, and Jayashankar M Swaminathan. "Managing Outsourced Software Projects: An Analysis of Project Performance and Customer Satisfaction.” Production and Operations Management 20, no. 4 (2011): 508-21.

[94] Nevado-Peña, Domingo, Víctor-Raúl López-Ruiz, and José-Luis Alfaro-Navarro. "Improving Quality of Life Perception with ICT Use and Technological Capacity in Europe.” Technological Forecasting and Social Change 148 (2019): 119734.

[95] Figueiredo, Lino, Isabel Jesus, J A Tenreiro Machado, Jose Rui Ferreira, and J L Martins De Carvalho. "Towards the Development of Intelligent Transportation Systems." In ITSC 2001. 2001 IEEE Intelligent Transportation Systems. Proceedings (Cat. No. 01TH8585), 1206-11. IEEE, 2001.

[96] Tayyaran, Mohammad R., and Ata M. Khan. "The Effects of Telecommuting and Intelligent Transportation Systems on Urban Development.” Journal of Urban Technology 10, no. 2 (2003): 87-100. doi:10.1080/1063073032000139714. 


\section{Appendix I}

Table A1. Primary Studies Related to the CSFs of ITS in the Literature.

\begin{tabular}{|c|c|c|}
\hline Study & Scope & Success Factors \\
\hline $\begin{array}{l}\text { Discovering the Implementation } \\
\text { Success Factors for IoT and Big } \\
\text { Data Analytics in Transportation } \\
\text { System [26] }\end{array}$ & $\begin{array}{l}\text { IoT and Big } \\
\text { Data Analytics } \\
\text { on ITS }\end{array}$ & $\begin{array}{l}\text { 1- Business } \\
\text { - Technology support; Electronic system adoption, Data analytics } \\
\text { - Innovation and Competitiveness; Cost strategy, Standardization, and quality } \\
\text { assurance, Business model } \\
\text { 2- Infrastructure } \\
\text { - Software; Augmented/Virtual reality } \\
\text { - Hardware; IoT/cloud computing software architecture, Autonomous vehicles } \\
\text { 3- Management and Administration } \\
\text { - Organizational culture; Employee reward system } \\
\text { - Data security and privacy governance; Customers' data privacy and security, } \\
\text { Cyber-attack counter measures and strategy, Research and development } \\
\text { initiative } \\
\text { - Data governance and legal framework; Data reusability, Data interoperability }\end{array}$ \\
\hline $\begin{array}{l}\text { Intelligent Transportations Systems: } \\
\text { Review of Current Challenges and } \\
\text { Success Factors: The Case of } \\
\text { Developing Countries [50] }\end{array}$ & $\begin{array}{l}\text { ITS Success } \\
\text { Factors on } \\
\text { Developing } \\
\text { Countries }\end{array}$ & $\begin{array}{l}\text { Infrastructure, Budget Restrictions, Interest among policy decision makers and } \\
\text { institutions, User and transport professional's awareness, Legal structures and } \\
\text { institutions, ITS Technology readiness and maturity }\end{array}$ \\
\hline
\end{tabular}

Table A2. Primary Studies Related to the CSFs of Blockchain in the Literature.

\begin{tabular}{l} 
Study \\
\hline $\begin{array}{l}\text { Evaluation of blockchain business success factors } \\
\text { using ahp [28] }\end{array}$ \\
Success factor of implementation blockchain \\
technology in pharmaceutical industry: A
\end{tabular}
literature review [13][52]

Success Factors of the Blockchain Adoption for Smart Manufacture [14]

Blockchain for Supply Chain Traceability: Business Requirements and Critical Success Factors [51]

Blockchain critical success factors for sustainable supply chain [15]

Key Success Factors of Blockchain Platform for Micro-enterprises [10]

A conceptual model of sustainable supply chain management in small and medium enterprises using blockchain technology [49]
Blockchain Technology in Smart Manufacture

Blockchain Technology in Supply Chain Management

Blockchain Technology in Sustainable Supply Chain Management

Blockchain Technology in Micro-Enterprises

Blockchain Technology in Sustainable Supply Chain Management on Small and Medium Enterprises
Decentralization, P2P Network, Transparency, Immutability, Anonymity, Smart Contract, Cryptography, Key Management, Scalability, Extensibility, Interoperability

Traceability, Track, Transparency, Trust, Real-time, Data provenance, Security, Private data, Cost savings, Serialization, Authentication, Auditability, Visibility, Efficiency, Automatically, Immutable, Consensus-driven, Reliable

Secure, Cost, Integrate, Trust, Automation, Traceable, Efficient, Risk, Real-time, Reliable, Sharing, Consensus, Decentralized, Distributed, Flexibility, Legal, Open, Safety, Transparent, Authentic, Accountable, Availability, Collaboration, Communication, Compliance, Density, Immutable, Knowledge Transfer, Permission less, Quality, Relation, Resilience, Scalability, Sustainability, Visibility

Technical Capabilities, Organizational Readiness, Other Cap. For Change, Goal Alignment, Partnership Trust, Stakeholder buy-in, Technological Maturity, Data Security, Technological feasibility, Information Capture, Operational Model, Internal Leadership within firm, External Leadership with stakeholders and in supply chain, Legal framework, Information stewardship

System robustness, Overall cost, Overall performance, Data safety and Decentralization, Accessibility, Laws and Policy, Smart system, Customer satisfaction, Reliable system, Documentation, Data management, Quality

Brand equity, Security anti-counterfeiting, Sales growth, Transparency and clear, Trust, Consensus mechanism, Traceability, Consistency, Tracking, Innovation management, International, Organization adaptation

Top Management support, Infrastructure, Financial Constraints, Planning \& Execution, Culture, Information communication Technology, Customer Acceptance, Supplier Acceptance, Government Support, Competition, External Stakeholders, People 
A TISM modeling of critical success factors of blockchain based cloud services [48]
User engagement, Industry collaboration, Rich ecosystem, Blockchain technology standardization, Regulatory clarity, Cost efficiency, Energy efficiency, Handling blockchain bloat, Miner incentives, Business case alignment to blockchain capability, Sidechains development, Blockchain talent pool, Leadership readiness for a decentralized consensus based technology, Technology investment and maturity, Trust on blockchain networks, Integration with other cloud services, Robust and mature Smart contracts platform, Blockchain Security, User control on data 